\title{
The Research on Network Measurement
}

\author{
Wenju Gao * \\ Department of Electronic Engineering, \\ Changchun Institute of Engineering Technology \\ Changchun, Jilin, China \\ gaowj913@sina.com \\ Yueou Ren \\ Department of Electronic Engineering, \\ Changchun Institute of Engineering Technology \\ Changchun, Jilin, China \\ renyueou@sina.com
}

\author{
Hongbo $\mathrm{Yu}$ \\ Department of Vehicle Engineering, \\ Changchun Institute of Engineering Technology \\ Changchun, Jilin, China \\ yuhongbo@sina.com \\ Qiuyan Li \\ Department of Electronic Engineering, \\ Changchun Institute of Engineering Technology \\ Changchun, Jilin, China \\ liqiuyan@sina.com
}

\begin{abstract}
Nowadays, the network and communication technology are developing rapidly, while the network Quality of Service (QoS) are often not satisfied, because of the overburdened network and the overloaded routers and switches. Therefore, regular measurement and evaluation to the relative parameters and indexes are absolutely necessary. In this paper, the functional module of network measurement system and the commonly used network measurement instruments are introduced; active measurement, passive measurement and the application of these two measurements are presented; the advantages and disadvantages of these measurements are analyzed.
\end{abstract}

Keywords- Network Measurement; Network Quality of Service $(Q o S)$; Network Measurement Instruments ; Active Measurement; Passive Measurement.

\section{INTRODUCTION}

To obtain stable and effective network environment, network bandwidth, packet loss rate, delay and timely estimate the effective management strategy is necessary. Network measurement is a series of operations to test and verify the characterization network performance indexes by software and hardware tools according to certain standard, method and technology ${ }^{[1]}$. Performance parameters are the tools to measure and describe the network performance. Network measurement aims at getting the performance parameters concerning data flow from real network environment, analyzing the state of network operation, finding out the causes of faults, and customizing relevant solutions. Network measurement includes the following:

\section{A. Performance assessment}

It is to observe the changes of network performance indexes under different network protocols (and their applications), to analyze network protocols and their "bottleneck" of application, to improve the existing protocol or develop new protocol. Usually, the network performance index is the key to performance assessment.

\section{B. Protocol debugging.}

Network measurement provides effective checking and testing for new protocol and application, and makes it consistent or compatible with the existing standard. Through protocol analys is towards the received data packets, the latest version of the network protocol and application is measured and problems are found, therefore, revision suggestions can be provided to developers.

\section{Fault diagnosis}

Some faults of network components will interfere with the operation of the whole network, like broadcast storm, address error, security attack, and illegal block length. Therefore, it is necessary to diagnose and analyze the faults, including locating the source of faults, log analysis, monitoring IP, IPX datagram traffic, realizing end to end testing in physicalsense or logical link.

\section{Characterization ofnetwork flow}

Network measurement is to analyze the original data on flows by using statistical techniques, and then extract the characteristics of the protocol or application. At present, the leading network flow monitors are $\mathrm{MRTG}^{[2]}$ and PRTG ${ }^{[3]}$, which use SNMP protocol to monitor data on flows and make charts according to data analys is ${ }^{[4]}$.

\section{THE FUNCTION MODULE OF NETWORK MEASUREMENT SYSTEM}

Usually, the structure of network measurement systemis like Fig. 1: 


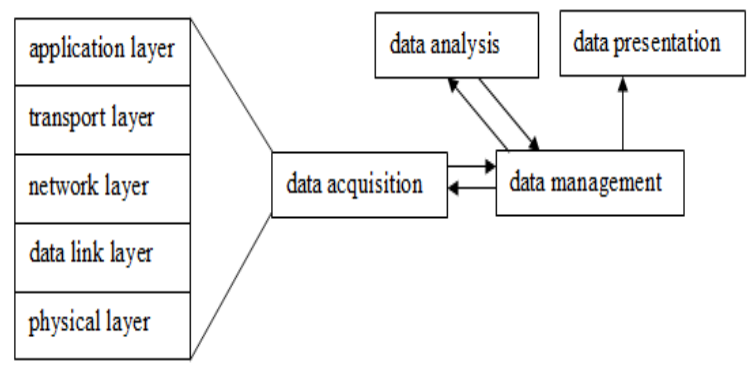

Figure 1. The function modules of network measurement system

There are four modules in the system: data acquisition, data presentation, data analysis, and data management. Data acquisition can be undertaken in any layer of the TCP/IP. The acquired data is submitted to data storage management, where the data is analyzed and restructured. Finally, the data is presented to users through data presentation.

\section{A. Data Acquisition}

The methods of data acquisition can be divided to active measurement and passive measurement. Active measurement is controllable, but it requires designing measurement grouping and testing process reasonably and effectively, and it will affect the tested network to some extent. Passive measurement requires choosing the location of measuring probe properly, but it will not affect the tested network.

The structure of the network is very complicated, so data acquisition is operated in the multi-channel environment. The acquisition methods are mainly: cycle sampling, synchronous sampling and interval sampling. Cycle sampling means acquiring data by connecting multi channels to $A / D$ cycle in some certain clock rate, using multiplexer. When time relationship among the channels is very important, cycle sampling will be necessary. While the effect of interval sampling is similar to that of synchronous sampling, because it can control scanning time and set the interval (microsecond or nanosecond level) between two samplings. In reality, interval sampling is usually applied, because of its high cost performance and fle xibility. Presently, WINCAP and SNIFFER are the two commonly used data acquisition tools ${ }^{[5]}$. WINCAP obtains the data packets sent and received by users through network interface card (NIC), monitors NIC and collects data packets passively (Fig. 2); SINFFER sets NIC as promiscuous mode, so as to receive every data packets transmitted through NIC.

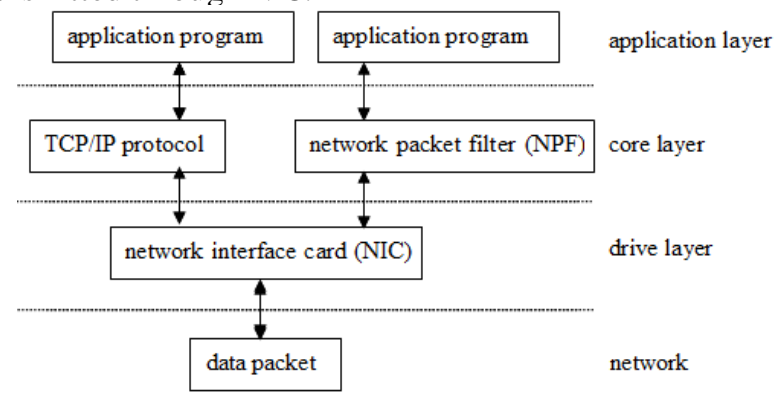

Figure 2. The structure and layers of WINCAP

\section{B. Data Analysis}

Data analysis is to research on the definition and computing of every index, and at the same time analyzes the different factors contained in the data, such as algorithm, the unit of measurement parameters and the sources of measurement error. Moreover, the network measurement errors and effects caused by using different methods and tools of under different environment should be considered. And all these factors should be described quantitatively. Presently, the relative performance indexes of network measurement are mainly included in the proposal and draft enacted by IPPM (IP Performance Metrics), such as packet loss rate, flow, connectedness, time-delay, link bandwidth and capacity, the average duration of connection, network topology structure and so on.

Presently, WIRESHARK/ETHEREAL and TCPDUMP are the leading network data analysis software ${ }^{[6]}$. The differences between them are as follow: WIRESHARK/ETHEREAL analyzes the acquired data by setting filtering rules and presents the protocol analysis structure through graphical interfaces. TCPDUMP is open source software, all of whose interfaces are open to public, so its extended function is strong. It is mainly applied to UNIX/LINUX system. It acquires network data packets, analyzes the fields in the packets and presents the results to users in the form of Trace files. Users can customize and program the trace file, and calculate the performance indexes

\section{Data Management}

The amount of raw data is huge, so its proper storage, maintenance and retrieval are indispensable. The formatting of data storage is convenient to the data analysis and retrieval. To solve the problem of data sharing, database technology is adopted generally. Meanwhile, to increase its flexibility, file storage can also be applied. To increase the data processing capacity, data is usually preprocessed before data analysis. Paper [7] introduces the data cleaning in the process of data preprocessing, which is to classify the data according to the requirement of the core data.

In the process of managing raw data, the most important step is to establish the retrieval strategy. In network measurement, when the requirements of different indexes are clear, retrieval strategy process can be structured according to the relevant parameter index. Relational database plays a very important role in measurement. It stores the acquired data according to the preset require ment, makes the data analyzed by application program, and restores the analyzed results to database, finally presents the data to user through user interface. Common relational database can not meet the requirement of performance and capacity, because of the high-speed network data flow. Therefore, RRDTOOL (Round Robin Database) can be designed to solve the problem ${ }^{[4]}$, which assigns the fixed sized space for data storage, with probe pointing to the address bit of the latest data. The data is stored in similar ring structure in the basis of time. If the data storage reaches its full capacity, new data will be written in covering the old data, so that the size of the data file remains the same, and the efficiency of data access will be increased. 


\section{Data Presentation}

Data presentation means to present the result of the network measurement clearly and intelligibly. Nowadays, monitoring tools such as MRTG, CACTI, PRTG ${ }^{[8]}$, present the measuring result to users in the form of flow chart drawn by the drawing engine of RRDTOOL. The parameters in the data of RRD are processed, then PNG images which are used to present on browser are created. In general, the content presented on PNG image is the acquired data.

\section{THE METHOD OF NETWORK MEASUREMENT}

Network measurement can be divided to active measurement and passive measurement according to different method of data acquisition.

Active measurement is to input a certain amount of data flow to the tested network, observe the response result, and analyze the relevant parameters of the data flow, so as to observe and measure the state and changes of the tested network $^{[9]}$. In this method of measurement, extra flow is input, and normal operation of the network will be affected to some extent. Therefore, the measuring result needs revising according to the added data flow so as to reflect the real operational state of the network. Passive measurement is to monitor and collect the network flow and data parameters so as to measure the network. Passive measurement can avoid the influence to the normal operation of the network, and the occurrence of measurement error, so that the performance of the network is affected to the minimum degree. Meanwhile, because of the relative fixed monitoring location, passive measurement mainly collects the data information with statistical significance, such as network flow, throughput, and time-delay, so as to analyze the state of network like the performance of link.

Active measurement can acquire the significant network indexes of link, such as the bottle neck bandwidth, bottleneck location, and topology change and so on ${ }^{[10]}$. Dovrolis and his partners use the technology of message pair to estimate the bandwidth of the link bottleneck in the probe gap model (PGM) . They analyze the relationship of interval between the probe message get in and out of the network, so as to get the end-to-end bottleneck bandwidth. The advantage of the model is simple algorithm and short measuring time. While there are also some problems such as, time expansion and time compression.

Jain $^{[13]}$ and his partners adopt SloPS (self-loading periodic stream) to measure the available bandwidth in probe rate model (PRM). The probe rate is similar to available bandwidth in congestion, therefore, PRM can change the probe rate of transmitting end, and observe the time-delay at the receiving end, from which the beginning time of the congestion can be determined, then available bandwidth can be estimated. The advantages of the model are its precision in measurement and availability in different states of network; while the disadvantages are that it will affect the state of network and the characteristic of flow, which leads to the instability of network, due to the relatively huge data input into the link.

$\mathrm{Hu}$ and his partners adopt recursive packet train (RPT) to locate the end-to-end link bottleneck. This technique actually combines the message queue and TRACEROUTE.
Transmitting end constructs and sends repeating message queue whose TTL increases progressively from 1 , so that message queue will return two consecutive ICMP message after each jump. According to the difference between the time of the two ICMP messages being received, bottleneck bandwidth from transmitting end to the route can be estimated, so that specific location of bottleneck of the whole link can be detected. Advantages of this method are relative less data input comparing to other algorithm, precise location, single-end detection, easy deploy and so on. There are also some disadvantages. To name some as follow: some parameters, such as the length, amount, and original infusion rate of the inputting packet length., which can affect location, can only be set according to the operators' experience, can't be adjusted according to the network environment.

In addition, Siekkinen ${ }^{[15]}$ and his partners, as well as Maier $^{[16]}$ and his partners research on the passive measurement deployed on IPS, which mainly concerns the flow model and the network usage of application program, obtains the throughput of RTT and household users. There is no favorable monitoring point in home-area network, so the real performance of the service that users get from the ISP can not be measured, for example, because of the speed limit of users' application program, the performance indexes from the ISP monitor can not reflect the real performance of the link. Han ${ }^{[17]}$ and his partners deploy passive measurement at user's side, which is to install monitoring software in users' computers. It can monitor and analyze the performance connected to the network from user's side conveniently, but is not suitable to monitor the performance of large-scale network. Meanwhile, it is difficult to obtain long-term, consistent measuring result, because users seldom run their computer continuously in the long-term. Sundares an and his partners set passive measurement at gateway, which can not only measure the performance of the link connected from ISP, but also can know the users' using state and the influence of users' behavior on the network performance. The method can be used to measure continuously in the long term, and to analyze the influence of different ISP flow model on throughput and time-delay.

Active measurement affects the operation of the tested network to some extent; therefore, there is some limitation on its application, while passive measurement almost does not affect the operation of the tested network, therefore, it is used widely, and is suitable to network monitor in the long term. If the characteristics of the network operation need depicting systematically, the two kinds of measurement should be used together and properly.

\section{THE REALIZATION OF NETWORK MEASUREMENT AND THE RELEVANT TOOLS}

\section{A. The Measurement Based on Flow}

The measurement based on flow is very similar to the recording of measuring details in the traditional network, both of whom regard flow as benchmark. The flow of network measurement can be defined as the collection of data packets which have the specific source address and destination address, transmit between source port and destination port, have fixed protocol type, and contain start time and end time. Take Surveyor of NSF as an example. 
It deploys customized measuring equipment at each node in measurement, and measures and record the one-way delay and packet loss rate on specific path in groups, by sending and receiving each other the active testing. The equipment of central database polls the measuring equipment every few minutes. If the measuring equipment gets new performance data, the new performance data will be uploaded to the central database. The system also analyze the asymmetry of the path, the asymmetry of packet loss rate, the asymmetry of time-delay, and the asymmetry of transmission among different areas ${ }^{[19]}$. CORA LREEF of CAIDA monitors the state of flow by the means of online monitoring, and then generates the flow measuring report by HTML report generation tool ${ }^{[20]}$. In addition, Test Traffic Measurement (TTM-RIS), IEPM and CFLOWD are all very good flow analyzing tools ${ }^{[20-22]}$.

\section{B. The Measurement Based on Network Interface, Interlinkage, and Node}

Every network element can be measured passively in network measurement. For example, NETRAMET realizes the measurement by sending ICMP packet to the tested interlinkage and contact, meanwhile collecting network interface data with SNMP as the agent. Its main measurement parameter is bandwidth[23]. IFFINDER of CAIDA measures each IP interface and realizes connection mainly by sending UDP packets to untapped ports [24]. IPERF developed by NLANR/DAST is used to measure TCP and UDP bandwidth, and meanwhile measure delay jitter and packet loss rate, by monitoring processes and client connection after staring the IPERF server[24]. Presently, NAI of NSF is the relatively comprehensive project in the foundational design of measurement. It collects the packet head data, active performance data and the data of the stability of network routing acquired by SNMP protocol[25]

\section{The Measurement Based on Node Pair}

In the traditional network transmission, every established connection contains a node pair, and can maintain data register for every node pair in every switch. But the similar measurement in IP network is a little more complicated, because IP is a datagram protocol, and there are many forwarding nodes, therefore, measurement data usually can't be obtained directly. To solve this problem, original data can be obtained through passive measurement and then be analyzed, so that the overall information of data flow can be obtained. But large amount of data need processing in this method, moreover, the tracking of flow between nodes will lost sometimes, because of the probable changes of routing configuration. To realize the measurement to node pair, a lot of tools are developed: PING is very basic. It can obtain many parameters, such as round-trip time delay between node pairs, packet loss rate, and connectivity. By sending ICMP packets to the other party of node pair, TRACERT/TRACEPATH detects the stopping location of data packet on network in the method of route tracking, so as to realize the measurement between node pairs. In addition, AUTOFOCUS developed by CAIDA collects data by tracking node pairs, and forms the visualized datagram and time line using RRDTOOL. PATGPING can also provide information about network delay and data loss to every router and link in path. Planet
Lab can realize the measurement between the node pairs ${ }^{[26-}$ 27].

\section{The Measurement Based on Path}

Path is the collection of links that IP packet passes through when it is transmitted from a source node to a target node. The measurement based on path main ly points to MPLS routing. Because MPLS can route network flow through relative fixed path, it can undertake measurement based on path, and develop access control mechanism based on flow, and track and estimate the specific service performance of transmission.

Presently, based on the path information collected from several source points to millions of object points, SKITTER discovers dynamically and tracks the internet topology structure adopting tomography, and draws the topology structure of the global internet by visualization technology. In the way of active measurement, NIMI developed by NSF and DA RPA deploys a large a mount of NPD (Network Probe Daemon) developed by Paxson on internet, and monitor the performance of internet path. NINI consists of probe, contact configuration center, data analysis center, and end of client measurement ${ }^{[29]}$. In addition, TRACEROUTE can measure the routing information of P2P. MTRACE can measure the multicast routing information. TRENO/CAP/CAPTRENO can measure the capacity of bulk transfer. ZING can measure one-way delay, round-trip delay and packet loss rate. MFLECT researches the characteristic of path by multicast. TRAFFICE/DISCARD can measure throughput. SPAND (Shared Passive Network Performance) measures the bandwidth with passive sharing measurement, and stores the result for sharing in the data center of network measurement performance in each domain. The computer in domain can obtain information from data center to estimate the performance, while not increase the load of the network ${ }^{[30]}$

\section{CONCLUSIONS}

Network measurement is significant to the development of network. The following research on network measurement lies in the cooperation of different measuring tools, constructing large-scale network measurement system, the visualization of measuring and analyzing result, network security problem caused by measurement, and development tendency of the network in the future.

\section{REFERENCES}

[1] Pei Changxing. "Contemporary Communication System and Network Measurement”. Beijing: Post \& Telecom Press, 2008.

[2] Oetiker T, Rand D. "The multirouter traffic grapher". [2011-0218]. http://oss.oetiker.ch/mrtg/,2012-09-10.

[3] Paessler "The network monitoring company. PRTG Manuals" [2012 - 08 - 10]. http://www.paessler.com/support/manuals/, 2012$09-10$.

[4] Shipway S. "Using MRT G with RRD tool and routers2" Cheshire Cat Publishing, 2011

[5] The WinPcap Team. "Winpcap documentation". http://www.winpcap.org/.

[6] Graderer G, Loschmidt P, Sauter T. "Rea-time enabled debugging for distributed systems" . //Proceedings of the ETFA 2008 IEEE International Conference. Hamburg: IEEE Press, 2008. 
[7] Zhang S C, Zhang C G, Yang Q. "Data preparation for data mining”. Applied Artificial Intelligence, 2003,17 (5-6): 375-381.

[8] The Cacti Group. "Official Cacti Documentation" . [2011-01-15]. http://docs.cacti.net/, 2012-05-10.

[9] Paxson A V. "A reactive measurement framework"//Proceedings of the 9th International Conference on Passive and Active Net work Measurement. Cleveland OH USA: Springer Publisher, 2008.

[10] Cunha I, Teixeira R, Veitch D. "Predicting and tracking internet path changes" // Proceedings of ACM SIGCOMM'11.Toronto: ACM Press, 2011.

[11] Dovrolis C, Ramanathan P, Moore D. "What do packet dispersion techniques measure" /Proceedings of IEEE INFOCOM'01. Anchorage (Alaska): IEEE Press, 2001

[12] Dovrolis C, Ramanathan P, Moore D. "Packet-dispersion techniques and a capacity-estimation methodology" . IEEE/ACM Transactions on Networking, 2004, 12(6): 963-977.

[13] Jain M, Dovrolis C. "Pathload: A measurement tool for end-to-end available bandwidth" /Proceedings of Passive and Active Measurements (PAM) Workshop, 2002. Fort Collins, Colorado, USA: CoteSeer, 2002.

[14] Hu N, Li L, Mao Z M. "Locating internet bottlenecks: algorithms, measurements, and implications" // Proceedings of ACM SIGCOMM'04. New York: ACM Press, 2004.

[15] Siekkinen M, Collange D, Urvoy-Keller G. "Performance lmitations of ADSL users: a case study" //8th International Conference, PAM 2007. Louvain-la-Neuve: Springer Berlin Heidelberg, 2007.

[16] Maier G, Feldmann A, Paxson V. "On dominant characteristics of residential broadband internet traffic" //Proceedings of the 9th ACM SIGCOMM Conference on Internet Measurement Conference. New York: ACM Press, 2009.

[17] Han D, Agarwala A, Andersen D G. "Mark-and-sweep: getting the inside scoop on neighborhood net works" /Proceedings of the 8th ACM SIGCOMM Conference on Internet Measurement Conference. New York: ACM Press, 2008.
[18] Sundaresan S, Donato W de, Teixeira R. "Broadband internet performance: a view from the gateway" /Proceedings of ACM SIGCOMM'11.Toronto: ACM Press, 2011.

[19] Calyam P, Lee C-G, Ekici E. "Orchestration of networkwide active measurements for supporting distributed computing applications" . IEEE Transactions on Computers, 2007, 56 (12): 1629-1642.

[20] The Cooperative Association for Internet Data Analysis. "CAIDA tools-overview of CAIDA software tools". [2011-12-15]. http://www.caida.org/tools/, 2012-05-11.

[21] RIPE (Reseaux IP Europeens) NCC. "Test traffic measurement service" [2012-06-16]. http://www.ripe.net/data-tools/stat s/ttm/testtraffic-measurement-service, 2012-06-21.

[22] Shriraml A, Murray M, Hyun Y. "Comparison of public end-to-end bandwidth estimation tools on high-speed links" //6th International Workshop, PAM 2005. Boston: Springer Berlin Heidelberg, 2005.

[23] Nlanr, Dast. Iperf. [2008-03-19]. http://iperf.sourceforge.net/, 2012-08-10.

[24] Govindam R, Tangmunarunkit H. "Heuristics for internet map discovery"//Proceedings of IEEE INFOCOM, 2000. Tel Aviv: IEEE Xplore, 2000.

[25] Gregor T Mc. "The nlamr network analysis infrastructure". IEEE Communications Magazine, 2000, 35(5): 122-128.

[26] Estan C, Savage S, Varghese G. "Automatically inferring patterns of resource consumption in net work traffic" //Proceedings of ACM Sigcomm, 2003. New York: ACM Press, 2003.

[27] Banerjee S, Grin T, Pias M. "The interdomain connectivity of planet lab nodes" /Proceedings of PAM, 2004. Antibes: Spinger Berlin Heidelberg, 2004.

[28] "The Cooperative Association for Internet Data Analysis". Skitter. [2011-12-15].http://www.caida.org/tools/measurement/skitter/, 2012-05-11.

[29] Paxson V, Adams A, Mathis M. "Experiences with NIMI" //SAINT 2000. Proceedings of Applications and the Internet (SAINT) Workshops. Nara: IEEE Conference Publications, 2002.

[30] Srinivasan S, Mark S, Katz R H. "SPAND: shared passive net work performance discovery" //USENIX. Proceedings of the 1st USIT S. Monterey: The Advanced Computing Systems Association, 1997. 